\title{
El complejo Bostrychietum: la flora de algas asociadas a las raíces del manglar en la costa pacífica colombiana
}

\author{
Enrique Peña-Salamanca \\ Universidad del Valle, Facultad de Ciencias Exactas y Naturales, Departamento de Biología \\ Artículo de posesión para el ingreso como miembro correspondiente de la \\ Academia Colombiana de Ciencias Exactas, Físicas y Naturales el 7 de junio de 2017 de 2017
}

\begin{abstract}
Resumen
El complejo Bostrychietum es conocido como una agrupación de algas asociada a las raíces de mangle en zonas tropicales y subtropicales. Se presenta una revisión de los aspectos taxonómicos, filogenéticos y ecofisiológicos de las especies del género Bostrychia distribuidas en el Pacífico colombiano. Los estudios basados en los caracteres taxonómicos y los análisis morfológicos del complejo concluyen que en el Pacífico colombiano habitan los siguientes morfotipos del género Bostrychia: B. calliptera y B. pinnata (morfo 1), B. moritziana y B. radicans (morfo 2), $B$. tenella (morfo 3), B. binderi (morfo 4). B. pinnata se considera sinonimia de $B$. calliptera, en tanto que $B$. moritziana y $B$. radicans conforman un grupo con morfología similar. La evaluación de genes nucleares, plastídicos y mitocondriales, así como la descripción complementaria de caracteres taxonómicos en Bostrychia, ha determinado que el complejo no es monofilético, es taxonómicamente indistinguible y está compuesto por tres linajes evolutivos. Desde el punto de vista ecofisiológico, las especies del complejo Bostrychetum están permanentemente expuestas a cambios extremos en el régimen lumínico, la salinidad y el nivel de inundación impuesto por la marea. $B$. calliptera y $C$. leprieurii registran fotosíntesis positiva en agua y en aire, lo que demuestran su capacidad para enfrentar los periodos de desecación durante la marea baja y explican el patrón de zonación y el ensamblaje algal en las raíces de mangle. Esta revisión plantea perspectivas de investigación que complementen los estudios taxonómicos y filogenéticos de las especies y evalúen su tolerancia al estrés ambiental propio del ambiente intermareal de los bosques de manglar. (C) 2017. Acad. Colomb. Cienc. Ex. Fis. Nat.
\end{abstract}

Palabras clave: Bostrychia; Manglar; Filogenia; Morfotipos; ADN plastidial RBC; Gen mitocondrial Cox 2-3, Fotosíntesis, Tolerancia al estrés.

The complex Bostrychetium: The algal flora associated to mangrove roots in the Pacific coast of Colombia

\begin{abstract}
The Bostrychietum complex is known as an algal assemblage associated with mangrove roots in tropical and subtropical areas. This review presents an analysis of the taxonomy, phylogeny and ecophysiology of Bostrychia species present in the Colombian Pacific Coast. Taxonomic and morphological studies have concluded that the following Bostrychia morphotypes are present: B. calliptera y B. pinnata (morpho 1), B. moritziana y B. radicans (morpho 2), B. tenella (morpho 3), B. binderi (morpho 4). B. pinnata is considered a synonym of $B$. calliptera, and $B$. moritziana and $B$. radicans are a group with differentiated morphology. The evaluation of nuclear, plastidic and mitochondrial genes, as well as complementary descriptions of Bostrychia taxonomic characters, has determined that the complex is non-monophyletic, that it is taxonomically indistinguishable and that it comprises three evolutionary lineages. From an ecophysiological point of view, Bostrychetum complex species are continuously exposed to extreme changes in the light regime, salinity and the tidal levels. B. calliptera and C. leprieurii exhibited positive photosynthesis activity in water and air. Such physiological response shows their capacity to survive to dessication periods during low tide and it explains the zonation pattern and the algal assamblages associated to mangrove roots. This review proposes research perspectives to complement species taxonomic and phylogenetic studies and to evaluate their resistance to intertidal environmental stress in mangrove forests. (C) 2017. Acad. Colomb. Cienc. Ex. Fis. Nat.
\end{abstract}

Key words: Bostrychia; Mangrove; Phylogeny, Morphotypes, Plastidic DNA RBC, Mitochondrial gene Cox 2-3, Photosynthesis, Stress tolerance.

Correspondencia:

Enrique Peña-Salamanca, enrique.pena@correounivalle.edu.co. Recibido: 31 de mayo de 2017. Aceptado: 19 de septiembre de 2017 


\section{Introducción}

El estudio de las algas en Colombia no ha tenido el mismo desarrollo que en otros países latinoamericanos como Brasil, Chile y México, donde la ficología presenta un mayor grado de madurez. Sin embargo, en el Pacífico colombiano se han logrado adelantar algunos trabajos orientados a la comprensión de diferentes aspectos bioecológicos y taxonómicos de la flora algológica, especialmente de aquellas asociadas a las raíces de manglar, uno de los principales biotopos de la zona intermareal en la línea costera del litoral Pacífico colombiano.

El complejo Bostrychietum es conocido como una agrupación de algas asociada a las raíces de mangle en zonas tropicales y subtropicales, compuesta principalmente por los géneros Bostrychia, Caloglossa, Catenella, Murrayella y Stictosiphonia (Chapman, 1976; King, et al., 1991; King \& Puttock, 1994). Los primeros estudios del complejo se orientaron a comprender la composición florística y la distribución de las especies (Post, 1963, Macnae, 1968). Recientemente, la importancia ecológica de estas comunidades de algas epífitas se ha evidenciado en su aporte significativo de biomasa para la producción de carbono del ecosistema (Laursen \& King, 2000) y por su rol como organismos bioindicadores (Peña, et al., 2005; Melville \& Pulkownik, 2006; Peña, et al., 2011). Se ha discutido su papel como productores primarios en el ecosistema de manglar (Burkholder \& Almodóvar, 1973; Rodríguez \& Stoner, 1990; Dawes, 1996), así como en las adaptaciones ecofisiológicas de algunas especies a las variaciones ambientales propias de la zona intermareal, especialmente a los cambios de salinidad y del ciclo de emersión e inmersión por efecto de la marea (Karsten, et al., 1994; Peña, et al., 1999; Karsten, et al., 2000; Peña, 2008). La mayoría de estos trabajos se han realizado en manglares de zonas subtropicales, pero es relativamente poca la atención que se ha dedicado a esta asociación en las áreas tropicales.

La mayoría de las especies que componen el complejo algal Bostrychietum pertenecen al orden Ceramiales del filo Rhodophyta, el cual comprende una gran variedad de especies de algas rojas con amplia distribución mundial (Guiry \& Guiry, 2016). Las especies están representadas principalmente por el género Bostrychia. Dentro de la familia Rhodomelaceae este grupo se diferencia principalmente por tener una disposición regular de las células de nivel, que corresponde a divisiones transversales de las células pericentrales que normalmente se encuentran en Rhodomeleaceae (Zuccarello \& West, 2006; Zuccarello \& West, 2011).

En la costa pacífica colombiana el género es muy importante por su contribución a la productividad primaria de ecosistemas costeros, y Bostrychia calliptera se ha determinado como un útil bioacumulador de metales y un indicador biológico de la contaminación en los estuarios (Peña, et al., 2005; Peña, et al., 2011). Además, es un recurso orgánico de un potencial excepcional por los compuestos con actividad biológica (Lenis, et al., 2007). Existen ambigüedades en la taxonomía del género Bostrychia (Rhodomelaceae) a nivel mundial, por lo que se ha empleado como un organismo modelo para la comprensión de la historia evolutiva de los órdenes agrupados en Florideophyceae.

Este trabajo presenta un análisis crítico de los estudios realizados sobre los aspectos taxonómicos, moleculares y ecofisiológicos de las especies asociadas al complejo Bostrychetium en la costa pacífica colombiana. Se discuten, asimismo, las adaptaciones de algunas especies a los ambientes propios del bosque de manglar y la perspectiva de investigaciones futuras para la comprensión de la taxonomía, la sistemática molecular y la ecofisiología de los ensamblajes de algas bénticas asociadas al manglar.

\section{Métodos}

Análisis filogenético de las especies del género Bostrychia. Para los estudios filogenéticos de las especies del complejo, se seleccionaron 48 individuos que agruparon diferentes morfotipos de $B$. calliptera/B. pinnata, $B$. radicans $/ B$. moritziana y $B$. tenella/B. binderi. Los ejemplares se obtuvieron mediante muestreos de campo realizados desde el 2010 hasta el 2015 en cuatro departamentos del Pacífico colombiano: Cauca (Isla Gorgona-LaAzufrada y La Ventana), Chocó (Ensenada de Utría y Río Juná), Nariño (Tumaco Estero Natal) y Valle del Cauca (Bahía de Buenaventura Estación Biología Marina, Estero Aguacate, Estero Hondo, Golfo de Tortugas, Piangüita, y Bahía Málaga - Juan Chaco, Chucheros y Ladrilleros), y se complementaron con otros individuos previamente recolectados y almacenados en la colección ficológica de referencia del Herbario CUVC "Luis Sigifredo Espinal-Tascón” y la Ficoteca del Laboratorio de Fisiología Vegetal de la Universidad del Valle.

La extracción del ADN genómico se hizo por maceración con nitrógeno líquido utilizando el estuche de extracción Wizard Genomic - Ref. A1120 (Promega, 2015). La determinación de la pureza y la cuantificación del ADN neto se estimaron en un espectrofotómetro Thermo Scientific NanoDrop 2000. La extracción del ADN de cada muestra se realizó con electroforesis en gel de agarosa al 0,8\% (p/v), y la corrida se hizo en TBE 0,5X (Tris-borato 0,045M; EDTA $0,001 \mathrm{M})$ a $100 \mathrm{~V}$ durante una hora. Los geles se tiñeron con bromuro de etidio $(\mathrm{BrE}-5 \mu \mathrm{g} / \mathrm{ml})$ y se visualizaron en transiluminador (TFP-MWL) de luz ultravioleta.

La amplificación del ADN se hizo con dos tipos de marcadores: con la región espaciadora del ADN mitocondrial $\operatorname{cox} 2$ - cox 3 y con el espaciador intergénico localizado entre la subunidad larga y la pequeña de la enzima ribulosa-1,5bisfosfato carboxilasa/oxigenasa (rubisco). En la reacción en cadena de la polimerasa (PCR) se emplearon las condiciones de amplificación descritas por Zuccarello, et al. (1999a). Los productos de la PCR se purificaron y se secuenciaron en el laboratorio de ficología de la Universidad de Louisiana, Estados Unidos, con la metodología descrita por Gavio \& Fredericq (2002). 
Las secuencias se editaron y ensamblaron en el programa Sequencher 4.1.4 (Gene Codes Corp., 2002). Se utilizó la herramienta BLAST de la plataforma del National Center for Biotechnology Information - NCBI para confirmar la amplificación exitosa de la región genómica de interés y establecer la identificación molecular de cada individuo. Los alineamientos para el análisis de las secuencias individuales y combinadas se hicieron en el programa Geneious 10.0.5, y se estimaron por máxima verosimilitud. La edición final se realizó en el programa FigTree 1.4.0 (Rambaut, 2009). En los análisis filogenéticos en el grupo interno se incluyeron secuencias adicionales del género Bostrychia y un grupo externo conformado por diferentes especies de macroalgas.

Evaluación de las respuestas fotosintéticas. Se tomaron en campo especímenes de las especies $B$. calliptera (Montagne) Montagne (Rhodomelaceae) y Caloglossa leprieurii (Montagne) G. Martens (Delesseriaceae) y se aclimataron durante 12 semanas en una cámara ambiental Sanyo (modelo Z82) a una temperatura promedio de $27{ }^{\circ} \mathrm{C}$, con un fotoperiodo de 12 horas de luz y 12 de oscuridad, en agua de mar enriquecida con medio de cultivo PES/2 (West, et al., 1993). En las especies del ensamblaje algal en el manglar se encontró suficiente biomasa para realizar los experimentos en laboratorio. Ambas especies se encontraron distribuidas a lo largo de la raíz del manglar, en poblaciones claramente diferenciadas en un patrón de zonación vertical (Peña, 1998). Las mediciones de la fotosíntesis en individuos sumergidos en agua se hicieron mediante el método de botellas claras y oscuras (Peña, 1998), y en los especímenes expuestos al aire mediante un analizador de gas infrarrojo IRGA (modelo Licor 6400) en una incubadora plexi-glass de un litro de capacidad. Todas las tasas de fotosíntesis se normalizaron como peso del alga en gramos por clorofila. Para la construcción de las curvas de luz y fotosíntesis (F-I), se utilizaron mallas de nylon de densidad neutra que simulaban diferentes intensidades lumínicas: 30, 60, 180, 350, 500, y $750 \mu \mathrm{mol} \cdot \mathrm{m}^{2} \cdot \mathrm{s}^{-1}$. Se evaluó la capacidad de recuperación fotosintética de las especies después de un periodo de desecación de dos horas, con el propósito de simular las condiciones en campo del ciclo mareal de emersión-inmersión de las poblaciones (Gylle, et al., 2009).

\section{Resultados}

Composición taxonómica del complejo Bostrychetium. El género Bostrychia pertenece a la familia Rhodomelaceae Harvey (1853), y actualmente es una de las diez familias que conforman el orden Ceramiales. La presencia de tricoblastos y el inicio del pericarpo antes de la fertilización son los caracteres taxonómicos propios de esta familia (Hommersand, 1963). La subfamilia Bostrychioide (De Toni, 1903; Hommersand, 1963) es un grupo distintivo dentro de Rhodomelaceae, conformado en la actualidad por dos géneros: Bostrychia y Stictosiphonia. La característica morfológica más evidente de identificación de esta subfamilia es la división transversal de las células pericentrales para formar dos o más células de nivel (Zuccarello \& West, 2006). Falkenberg (1901) fue el primero en revisar la taxonomía del género Bostrychia con base en el análisis del número de células de nivel derivadas de las células pericentrales, y quien separó el género Stictosiphonia del primero.

La designación de especies del género Bostrychia por taxonomía clásica ha demostrado ser muy compleja, entre otras razones debido a la plasticidad fenotípica inducida por el hábitat y la diversidad críptica del género (Zuccarello \& West, 2003; Zuccarello, et al., 2015). Por lo tanto, para abordar el problema del status taxonómico de las especies de Bostrychia, es necesario integrar los datos moleculares y los morfológicos para facilitar la comprensión de los factores que inciden en la composición y distribución de este grupo a escala global (Zuccarello, et al., 2011). En algunos trabajos hechos desde un enfoque florístico se ha dado cuenta de la dificultad de la identificación morfológica clásica de algunas especies del género Bostrychia presentes en la costa pacífica colombiana (Bula-Meyer, 1990; Bula-Meyer, 1995; Peña, 2008; Murillo \& Peña, 2013). En estos trabajos se ha evidenciado la desactualización de las pocas claves taxonómicas disponibles para macroalgas de Colombia y la poca atención que se le ha dedicado a esta asociación algal en las áreas tropicales. Los trabajos pioneros de Bula \& Schnetter (1982) establecieron que el número de especies de macroalgas en el Pacífico colombiano superaba los 150 taxones. Por el contrario, en la costa atlántica colombiana, los registros indican una diversidad algal más alta. Díaz \& Díaz (2003) reportaron un total de 565 taxones de algas marinas bentónicas mediante nuevas recolecciones en diferentes regiones costeras del Caribe colombiano.

En el Pacífico colombiano se ha registrado la presencia de varias especies de los géneros Bostrychia, Caloglossa, Catenella y Murrayella (Schnetter \& Bula-Meyer, 1982; Baos \& Morales, 2007; Peña, 2008; Murillo \& Peña, 2013; Peña \& Marín, 2017). El género Stictosiphonia no presenta registros en esta región geográfica. Cabe aclarar que, a pesar de los esfuerzos de muestreo realizados, aún hoy muchas zonas de la costa norte del Pacifico, como el Chocó, que no se han explorado y hacerlo podría aumentar el número de registros e inventarios de la ficoflora presente en Colombia. En la Tabla 1 se registra el listado taxonómico actual de las especies de macroalgas para el Pacifico colombiano.

Sistemática y filogenética del complejo Bostrychetium. El desarrollo de la biología molecular ha permitido avanzar en la comprensión de la historia evolutiva de las algas. Sus métodos, integrados a la taxonomía clásica, reflejan con mayor precisión la evolución de estos organismos (Markmann \& Tautz, 2005; Zuccarello \& West, 2011; Hu \& Fraser, 2016; Zuccarello \& West, 2016). La taxonomía molecular asistida ha sido una herramienta apropiada para avanzar en la comprensión de la variación morfológica (plasticidad) y en el establecimiento del estatus taxonómico (caracteres diagnósticos) de grupos algales cuyos caracteres morfológicos por sí solos no resuelven la ambigüedad 
Tabla 1. Listado taxonómico de macroalgas en el pacífico colombiano

\begin{tabular}{|c|c|c|}
\hline Género (Autoridad taxonómica) & Listado especies & Distribución pacífico colombiano \\
\hline \multicolumn{3}{|l|}{ RHODOPHYTA } \\
\hline Bostrychia (Montagne, 1842) & $\begin{array}{l}\text { Bostrychia binderi } \\
\text { Bostrychia calliptera } \\
\text { Bostrychia moritziana } \\
\text { Bostrychia pinnata } \\
\text { Bostrychia radicans } \\
\text { Bostrychia tenella }\end{array}$ & $\begin{array}{l}\text { Bahía Málaga, Buenaventura, Delta del Río } \\
\text { Dagua, Guapi, Panamá, Tumaco, Utría }\end{array}$ \\
\hline Caloglossa (Harvey) G.Martens, 1869) & $\begin{array}{l}\text { Caloglossa beccarii } \\
\text { Caloglossa leprieurii } \\
\text { Caloglossa ogasawarensis } \\
\text { Caloglossa stipitata }\end{array}$ & $\begin{array}{l}\text { Bahía Málaga, Buenaventura, Delta del Río } \\
\text { Dagua, Tumaco, Utría }\end{array}$ \\
\hline Callithamnion (Lyngbye, 1819) & Callithamnion rupinicola & Tumaco \\
\hline Catenella (Greville, 1830) & $\begin{array}{l}\text { Catenella caespitosa } \\
\text { Catenella impudica }\end{array}$ & $\begin{array}{l}\text { Bahía Málaga, Buenaventura, Delta del Río } \\
\text { Dagua, Tumaco }\end{array}$ \\
\hline Ceramium (Roth, 1797) & $\begin{array}{l}\text { Ceramium procumbens } \\
\text { Ceramium sinicola }\end{array}$ & Isla Gorgona, Tumaco \\
\hline Gelidiopsis (F. Schmitz, 1895). & Gelidiopsis spp. & Isla Gorgona. \\
\hline Gelidium (J.V. Lamouroux, 1813) & $\begin{array}{l}\text { Gelidium isabelae } \\
\text { Gelidium pusillum } \\
\text { Gelidium pusillum var. pulvinatum }\end{array}$ & $\begin{array}{l}\text { Buenaventura, Delta del Río Dagua, Isla } \\
\text { Gorgona, Tumaco }\end{array}$ \\
\hline Gracilaria (Greville, 1830) & $\begin{array}{l}\text { Gracilaria blodgettii } \\
\text { Gracilaria sjoestedtii } \\
\text { Gracilaria } \text { spp. }\end{array}$ & Isla Gorgona, Tumaco \\
\hline Heterosiphonia (Montagne, 1842) & Heterosiphonia $s p$ & Isla Gorgona \\
\hline Hypnea (J.V. Lamouroux, 1813) & $\begin{array}{l}\text { Hypnea pannosa } \\
\text { Hypnea valentiae }\end{array}$ & Isla Gorgona \\
\hline Jania (J.V. Lamouroux, 1812) & Jania spp. & Isla Gorgona \\
\hline Liagora (J.V.Lamouroux, 1812) & Liagora spp. & Isla Gorgona \\
\hline Lithothamnium (R.A. Philippi, 1837) & Lithothamnium spp. & Isla Gorgona \\
\hline Lithophyllum (Philippi, 1837) & Lithophyllum spp. & Isla Gorgona \\
\hline Murrayella (F. Schmitz, 1893). & Murrayella periclados & Tumaco \\
\hline Polysiphonia (Greville, 1823). & $\begin{array}{l}\text { Polysiphonia flaccidissima } \\
\text { Polysiphonia howei }\end{array}$ & Isla Gorgona, Tumaco \\
\hline \multicolumn{3}{|l|}{ HETEROKONTOPHYTA } \\
\hline Dictyopteris (J.V. Lamouroux, 1809) & Dictyopteris spp. & Isla Gorgona \\
\hline Dictyota (J.V. Lamouroux, 1809) & $\begin{array}{l}\text { Dictyota divaricata } \\
\text { Dictyota adnata }\end{array}$ & Isla Gorgona, Tumaco \\
\hline Colpomenia (Derbès \& Solier, 1851) & Colpomenia sinuosa & Isla Gorgona. \\
\hline Lobophora (J. Agardh, 1894) & Lobophora variegata & Isla Gorgona \\
\hline Padina (Adanson, 1763) & Padina crispata & Isla Gorgona, Tumaco \\
\hline \multicolumn{3}{|l|}{ CHAROPHYTA } \\
\hline Nitella (C. Agardh, 1824) & Nitella spp. & Isla Gorgona \\
\hline \multicolumn{3}{|l|}{ CHLOROPHYTA } \\
\hline Acetabularia (J.V.Lamouroux, 1812) & Acetabularia spp. & Isla Gorgona \\
\hline Avrainvillea (Decaisne, 1842) & Avrainvillea spp. & Isla Gorgona \\
\hline Boodleopsis (A.Gepp \& E.S.Gepp, 1911) & Boodleopsis verticillata & Buenaventura, Delta del Río Dagua, Tumaco \\
\hline
\end{tabular}




\begin{tabular}{lll}
\hline Género (Autoridad taxonómica) & Listado especies & Distribución pacífico colombiano \\
\hline CHLOROPHYTA & & \\
\hline Caulerpa (J.V.Lamouroux, 1809) & $\begin{array}{l}\text { Caulerpa racemosa } \\
\text { Caulerpa sertularioides }\end{array}$ & Isla Gorgona, Tumaco \\
\hline Chaetomorpha (Kützing, 1845) & Chaetomorpha antennina & Buenaventura, Tumaco \\
\hline Cladophora (Kützing, 1843) & Cladophora albida & Isla Gorgona \\
\hline Cladophoropsis (Børgesen, 1905) & Cladophoropsis membranacea & Buenaventura, Isla Gorgona, Tumaco \\
\hline Derbesia (Solier, 1846) & Derbesia spp. & Isla Gorgona \\
\hline Dictyosphaeria (Decaisne, 1842) & Dictyosphaeria cavernosa & Isla Gorgona \\
\hline Enteromorpha (H.F. Link, 1820) & Enteromorpha flexuosa & Tumaco \\
\hline Gayralia (K.L. Vinogradova, 1969) & Gayralia oxisperma & Tumaco \\
\hline Rhizoclonium (Kützing, 1843) & Rhizoclonium riparium & Bahía Málaga, Buenaventura, Tumaco \\
\hline Udotea (J.V.Lamouroux, 1812) & Udotea sp & Isla Gorgona \\
\hline Ulva (Linnaeus, 1753) & Ulva spp. & Tumaco \\
\hline Ulvaria (Ruprecht, 1850) & Ulvaria oxysperma & Isla Gorgona \\
\hline Ventricaria (J.L. Olsen \& J.A.West, 1988) & Ventricaria spp. & Isla Gorgona \\
\hline
\end{tabular}

taxonómica, las sinonimias, ni las relaciones filogenéticas (Cianciola, et al., 2010; Ruggiero, et al., 2015; Zuccarello, et al., 2015). Es así como se ha podido establecer que las especies crípticas son muy similares en cuanto a su apariencia morfológica pero distantes a nivel genético (Mayr, 1982). Por lo tanto, el avance integral de la sistemática molecular en macroalgas es determinante en la comprensión de los procesos de distribución y especiación de la flora marina tropical.

El problema biológico de especiación críptica en el complejo Bostrychetium fue abordado inicialmente por Zuccarello \& West (2003), Zuccarello, et al. (2006), Muangmai, et al. (2015), Zuccarello, et al. (2015), y Saengkaew, et al. (2016). La falta de caracteres diagnósticos adecuados o evidentes en algunos grupos algales, como el caso del género Bostrychia, es un indicador del alto grado de plasticidad fenotípica observado en muchas poblaciones y en diferentes hábitats. Este problema es notable en la clase Florideophyceae, categoría taxonómica considerada como monofilética y que constituye el grupo de algas rojas más abundante y diverso (Zuccarello \& West, 2003; Zuccarello, et al. 2011; Yang, et al., 2016).

El uso de los caracteres taxonómicos tradicionales para el estudio morfológico de las especies del género Bostrychia ha dificultado la aclaración del estatus taxonómico de las especies reportadas en el Pacífico colombiano, y ello justifica el uso de técnicas moleculares para su identificación. En los trabajos sobre este grupo realizados desde una perspectiva molecular se ha concluido que en el Pacífico colombiano habitan seis morfotipos del género Bostrychia (Hernández-Contreras, 2017), cuatro de los cuales son similares a los propuestos por Zucarello \& West (2011) (Figura 1).
Es importante resaltar que aún no está resuelta la naturaleza críptica en el morfotipo $B$. radicans/B. moritziana, cuya diferencia morfológica principal radica en la presencia de ramas laterales monosifónicas en $B$. moritziana, ya que estos caracteres morfológicos no han sido suficientes para identificar las especies presentes en el Pacífico colombiano. Zuccarello, et al. (1999b) y Zuccarello \& West (2003) determinaron mediante la reconstrucción filogenética de secuencias de ADN plastidial y mitocondrial que el complejo Bostrychia radicans/B. moritziana se compone de siete linajes evolutivos distintos en individuos hallados a grandes distancias geográficas. Estos linajes indiferenciados morfológicamente pero genéticamente distintos han cuestionado la definición de especie para el grupo Bostrychetium.

Los análisis filogenéticos recientes han determinado que el complejo no es monofilético, es taxonómicamente indistinguible y está compuesto por tres linajes evolutivos, considerándose $B$. pinnata como sinonimia de $B$. calliptera.

La caracterización molecular de los morfotipos identificados como del género Bostrychia en el Pacifico colombiano a partir de secuencias del ADN del plastidio y de la mitocondria, indican que $B$. pinnata es sinonimia de $B$. calliptera (Figura 2). Los morfotipos $B$. radicans y $B$. moritziana se consideran especies crípticas. Sin embargo, los morfotipos reportados de $B$. radicans y $B$. tenella se han propuesto como una asociación a nivel genético particular del Pacífico colombiano. La filogenia del gen plastídico $r b c$ (Figura 3) está conformada por dos grupos: el grupo 1 (G1) compuesto por $B$. radicans, $B$. moritziana, B. anomala y $B$. bispora, y el grupo 2 (G2) integrado por individuos de $B$. intricata, B. arbuscula, B. gracilis, B. radicosa, el complejo 
A

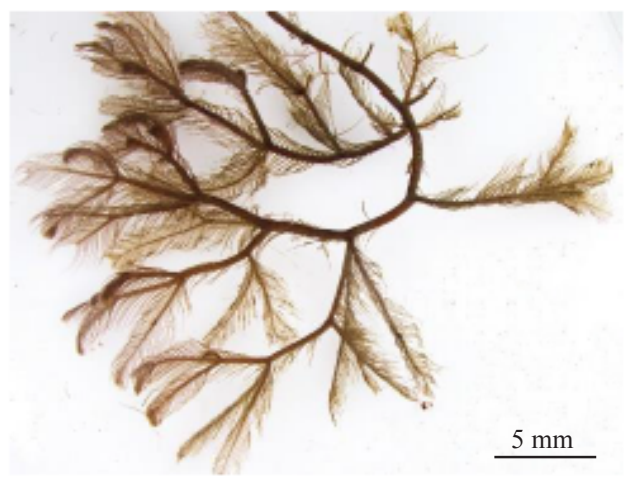

C

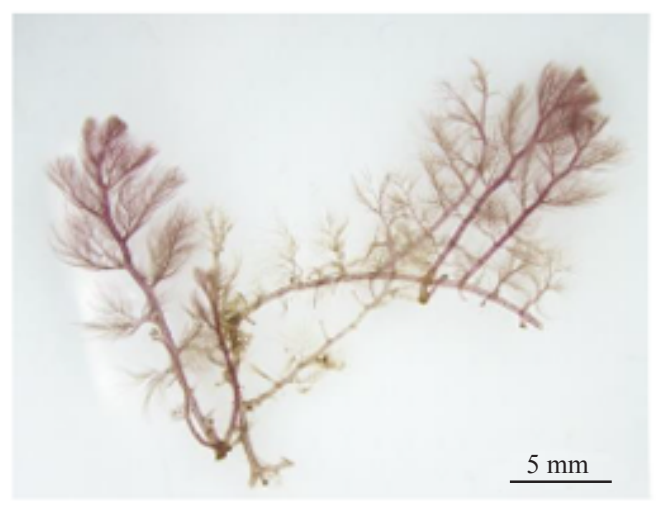

E

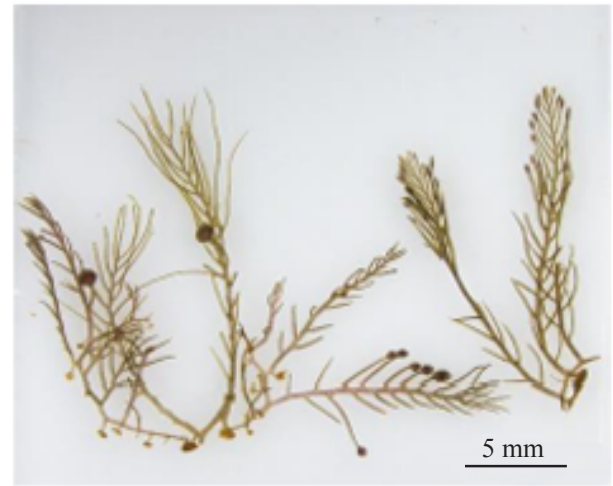

B

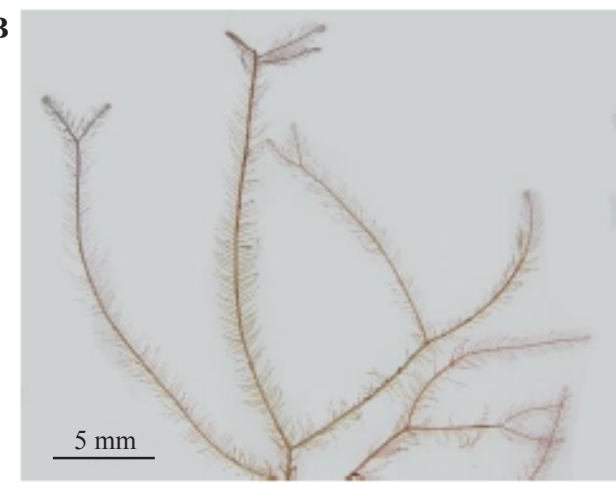

D

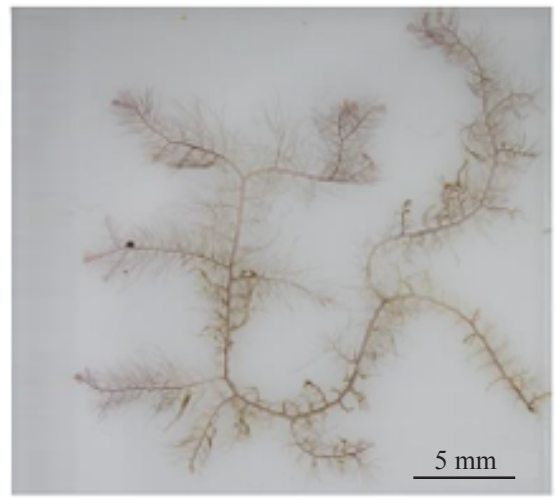

$\mathbf{F}$

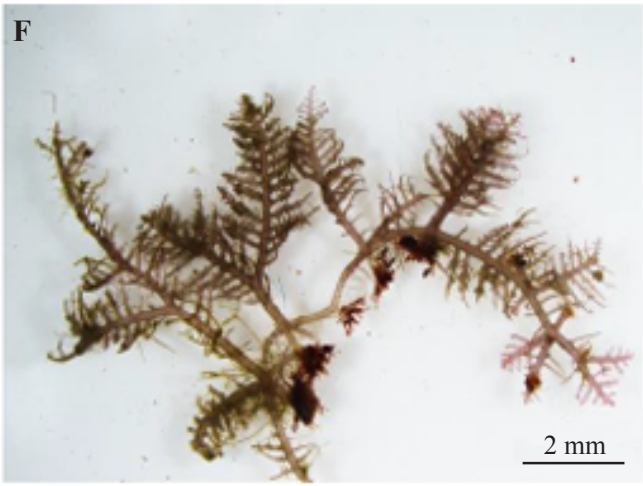

Figura 1. Morfotipos del género Bostrychia identificados en el Pacífico colombiano. A. Bostrychia calliptera y B. B. pinnata. C. $B$. radicans y D. B. moritziana. Morfotipos únicos de: E. B. tenella y F. B. binderi.

B. calliptera / B. pinnata, y un individuo de Bostrychia sp. Los análisis de máxima verosimilitud para el cox 2-3 y el $r b c$ arrojaron topologías diferentes en cada gen, sin embargo, el clado conformado por los individuos de $B$. calliptera/B. pinnata del Pacífico colombiano se resolvió como monofilético en los dos genes con buenos porcentajes de nodo: $100 \%$ para el gen mitocondrial y $82 \%$ para el plastídico. En general, las filogenias muestran dos clados de Bostrychia, y $B$. calliptera, B. pinnata, B. radicans, B. moritziana se consideran especies crípticas, en tanto que $B$. tenella es críptica con respecto a $B$. radicans.

Aunque los resultados de las filogenias registradas evidencian la presencia de linajes genéticos entre los morfotipos estudiados, aún quedan por establecer diferencias suficientes en los caracteres morfológicos para entender el status taxonómico de las especies del complejo. Sin embargo, los datos genéticos respaldan la hipótesis planteada por Zuccarello \& West (2011) que considera B. calliptera y B. pinnata como una misma especie.

Ecofisiología de las especies del complejo Bostrychetium. En el Pacífico colombiano el complejo Bostrychetium converge, por lo general, en un mismo nicho ecológico en los hábitats de manglar y crece estratificado en las raíces de árboles de mangle como Pelliciera rhizophorae, Rhizophora mangle y en neumatóforos como Avicennia germinans (Peña, 2008). También se ha reportado el género Bostrychia en arrecifes de coral en Isla Gorgona (Murillo \& Peña, 2013). El complejo es importante en la composición de 


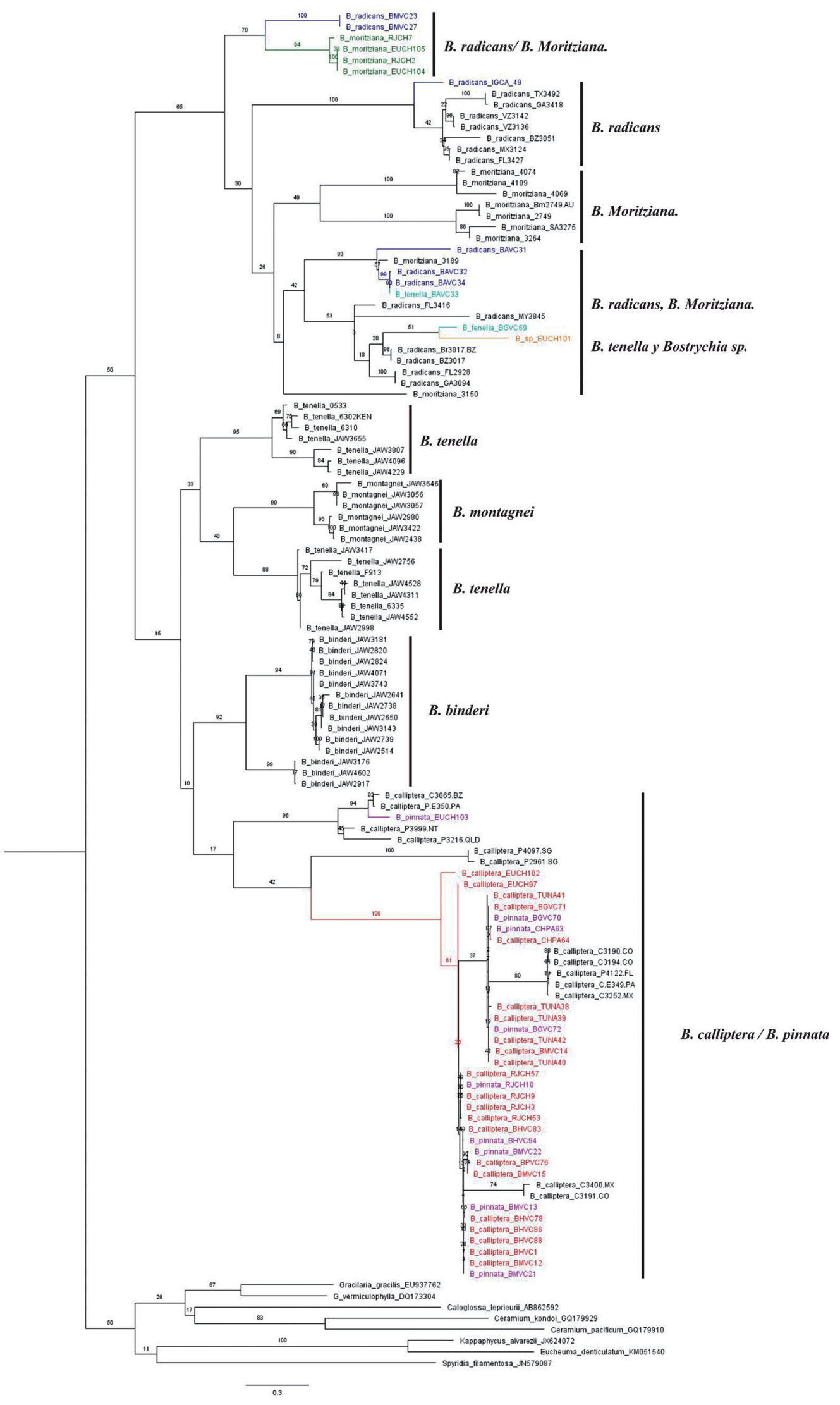

Figura 2. Filogenia de Bostrychia corrida en máxima verosimilitud con secuencias del gen espaciador cox 2-3. Las muestras en color son del Pacífico colombiano, y las demás provienen de Genbank. 


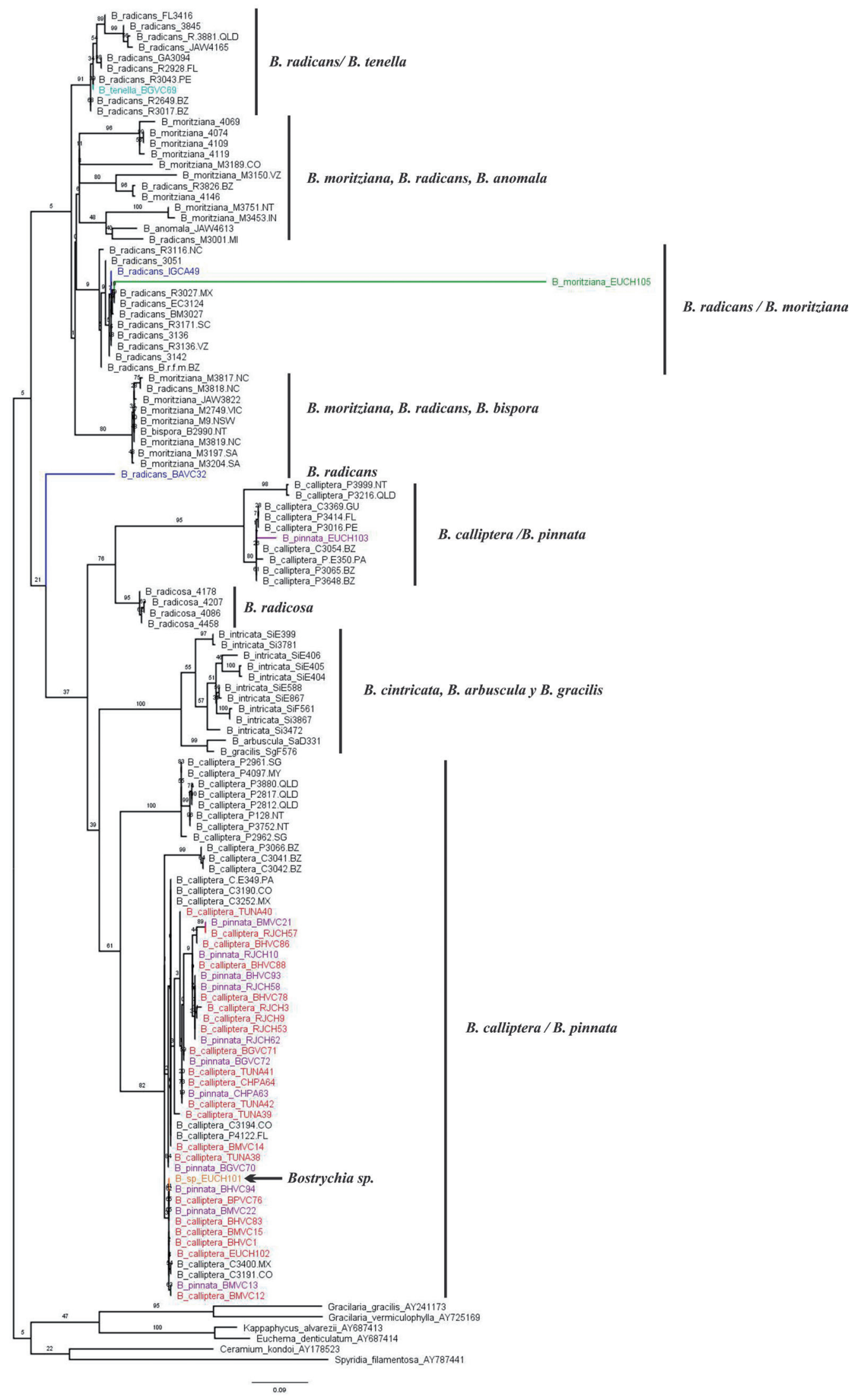

Figura 3. Filogenia basada en la máxima verosimilitud de las secuencias del gen espaciador rbc de especies del género Bostrychia $(-\operatorname{lnL}=4912.8251)$ 
las redes tróficas de los ecosistemas acuáticos debido a su significativo aporte de biomasa en la producción de $\mathrm{CO}_{2}$ y por el papel de sus especies como organismos bioindicadores (Peña, et al., 1999, Laursen \& King, 2000; West, et al., 2013).

Peña (2008) evaluó la composición de la biomasa algal de las especies dominantes del complejo en áreas de manglar en la bahía de Buenaventura y concluyó que $B$. calliptera (Montagne) Montagne aportaba un $32 \%$ de la biomasa total de algas en la raíz, mientras que Catenella impudica (Montagne) J. Agardh aportaba un $18 \%$ y C. leprieurii (Montagne) J. Agardh un $12 \%$. La dominancia de ciertas especies de algas en estos ambientes indica que la flora algal en estas zonas de manglar es relativamente homogénea y presenta poca diversidad de especies (Peña, et al., 2005).

La evaluación de las respuestas fisiológicas de la planta frente a la variabilidad de los parámetros ambientales es clave para entender la distribución de los ensamblajes algales en un determinado ambiente (Larcher, 1989). En el caso de las algas asociadas al manglar, la variabilidad de la salinidad, la desecación en marea baja y las variaciones de la intensidad lumínica imponen restricciones en su distribución y zonación (Peña, 1998). Las mediciones de la actividad fotosintética en agua y en aire de $B$. calliptera y $C$. leprieurii han arrojado tasas positivas, lo cual demuestra su capacidad adaptativa a ambientes intermareales hostiles, como el caso de los planos de lodo del manglar (Abe, et al., 2001; Peña, et al., 2005; Leprince \& Buitink, 2010).

En ambas especies la fotosíntesis neta fue mayor en agua que en aire. Sin embargo, la tasa de fotosíntesis máxima $\left(\mathrm{F}_{\max }\right)$ fue significativamente mayor en $B$. calliptera que en $C$.

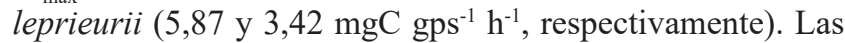
curvas de fotosíntesis e irradiancia (F-I) para ambas especies sugieren una adaptación a hábitats sombreados (Figura 4 a, b), con base en los puntos de compensación de luz en agua $\mathrm{y}$ en aire, los cuales estuvieron por debajo del promedio de intensidad de la luz de $25 \mu \mathrm{mol} \cdot \mathrm{m}^{-2} \mathrm{~s}^{-1}$. Estos resultados coinciden con los modelos de Parker (1974) y Jasby \& Platt (1980), ampliamente utilizados para comprender la dinámica fotosintética de plantas de sotobosque y de poblaciones naturales de algas Kelp en latitudes sublitorales (Coutinho \& Zingmark, 1987, Dinakar, et al., 2012). Durante la marea alta, la irradiancia en la columna de agua se ve muy reducida por la resuspensión de sedimentos y la gran turbidez. La reducción del ambiente lumínico en el manglar implica una adaptación del aparato fotosintético de las algas, lo que se demuestra con los valores de los parámetros fotosintéticos de las curvas de F-I de $B$. calliptera y $C$. leprieurii. Resultados similares se han observado en otras especies como Ulva curvata (Vergara, et al., 1997), Mastocarpus stellatus (Collén \& Davison,1999) y Mazzaella laminarioides (Contreras-Porcia, et al., 2011; Flórez-Molina, et al., 2014). Las especies de ambientes intermareales expuestas regularmente a periodos de desecación han desarrollado estrategias de protección y adaptación a la luz que les permiten manejar el estrés lumínico e hídrico impuesto por el régimen mareal (Lobban \& Harrison, 1994; Peña, 1998, Moore, et al., 2009, Flórez-Molina, et al., 2014).

Se evidenció un aumento de la actividad fotosintética en $B$. calliptera en condiciones de 15 a $25 \%$ de desecación, y las tasas disminuyeron a partir de ese porcentaje con la disminución del contenido de agua. En C. leprieurii la desecación influyó negativamente en las tasas fotosintéticas, las cuales disminuyeron significativamente con la disminución del contenido de agua, aunque por debajo de $28 \%$ del contenido relativo de agua, los individuos de esta especie registraron actividad respiratoria. Para el caso de $B$. calliptera, los individuos mostraron $100 \%$ de recuperación después de 45 minutos, mientras que en $C$. leprieurii la recuperación de $100 \%$ solo se dio después de 105 minutos. Estos resultados muestran la clara evidencia de la capacidad de $B$. calliptera para manejar el estrés hídrico y adaptarse a mayores tiempos de desecación durante la marea baja. Estas estrategias fisiológicas de las especies del complejo Bostrychetium explican en gran parte su patrón de zonación en las raíces de mangle. En efecto, esta especie registró los valores más altos de biomasa en la parte alta de la raíz, por encima 30,7 $\pm 10,8 \mathrm{~g} \mathrm{~m}^{-2}$ (Peña, 2008). La diferencia en la distribución vertical de las macroalgas epífitas y su relación

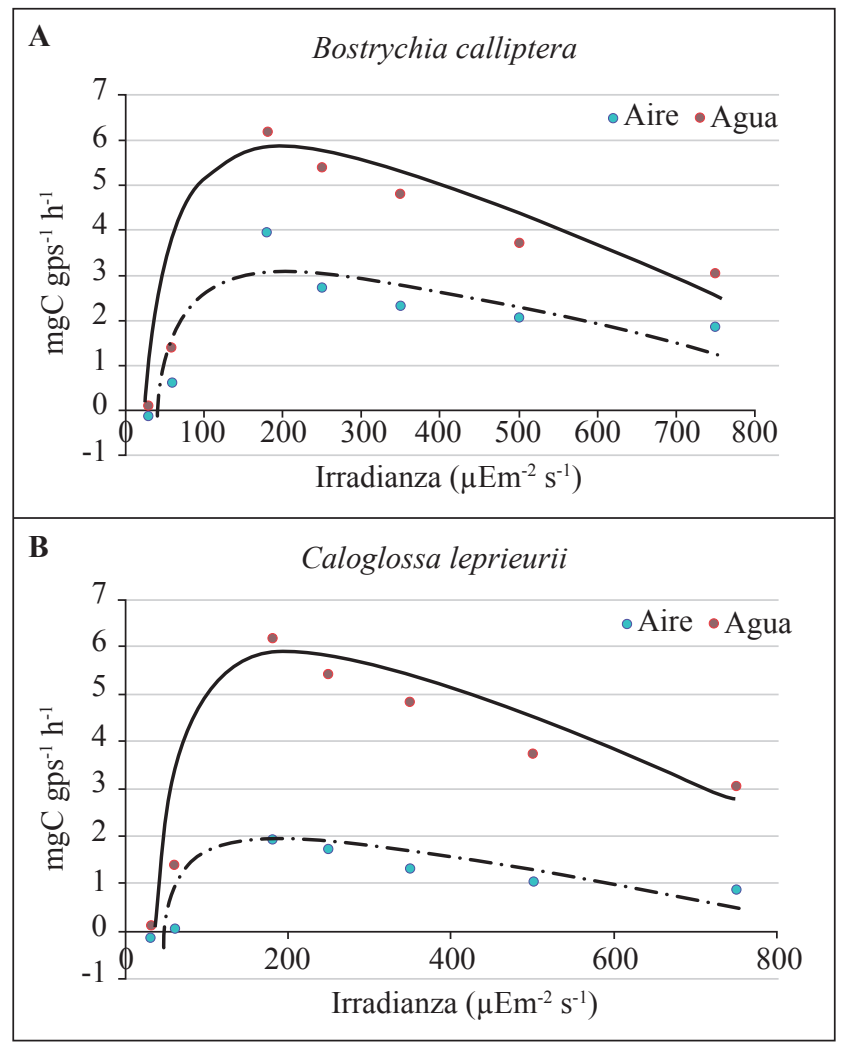

Figura 4. Curvas de fotosíntesis-irradiancia de: A) Bostrychia calliptera y B) Caloglossa leprieurii en agua y en aire. Los datos se ajustaron al modelo de fotoinhibición de Jasbyy \& Platt (1980). Las barras verticales representan un error estándar de $\pm 1(\mathrm{n}=5)$. 
con la actividad fotosintética a lo largo de las raíces de Rhizophora y neumatóforos de Avicennia se ha observada en otros manglares (Tanaka \& Chihara, 1987; Peña, 1998; Karsten, et al., 2000; Kim \& Garbary, 2007). Se ha detectado la producción de compuestos peptídicos de la familia de la micosporina-glicina y de pigmentos específicos que absorben luz en especies como B. radicans, C. impudica y C. leprieurii (Karsten, et al., 1998, 2000). Este tipo de cromóforos son comunes en algas que colonizan ambientes con alta variabilidad lumínica, como el caso de las áreas de manglar, las zonas rocosas intermareales, y los planos de lodo del mesolitoral en zonas estuarinas, cuyas condiciones oceanográficas y biofísicas se han considerado ambientes "estresantes" para el ensamblaje de la flora algal asociada (Larcher, 1989; Skene, 2004). Las adaptaciones de ciertas especies de estos ensamblajes, como el caso de B. calliptera, se han estudiado ampliamente y se la ha propuesto como planta modelo en estudios de estrés algal y por las propiedades de sus metabolitos (Zucarello \& West, 2011). Yarish, et al. (2004) propusieron el uso de Bostrychia como alga modelo para estudios de bioacumulación de metales pesados. Peña, et al. (2011) examinaron la dinámica de acumulación del cromo en consorcios de algas y bacterias para procesos de asimilación y eliminación de metales. Estos resultados indican que las especies del género Bostrychia pueden ser usadas en procesos de biorremediación para la solución de problemas ambientales de contaminación marina.

\section{Conclusiones}

Mediante el estudio de los caracteres taxonómicos y los análisis morfológicos de las especies del complejo Bostrychetium que habitan en el Pacífico colombiano se pudo confirmar la presencia de varios morfotipos, los cuales se resumen así: $B$. calliptera y $B$. pinnata (morfo 1 ), $B$. moritziana y $B$. radicans (morfo 2 ), $B$. tenella (morfo 3 ), $B$. binderi (morfo 4); $B$. pinnata se considera sinonimia de $B$. calliptera, en tanto que Bostrychia moritziana y $B$. radicans conforman un grupo con morfología similar. Bostrychia tenella y Bostrychia binderi se establecieron como dos especies diferenciadas.

En estudios recientes de evaluación de genes nucleares, plastídicos y mitocondriales, así como de descripción complementaria de los caracteres taxonómicos en Bostrychia, se ha determinado que el complejo no es monofilético, es taxonómicamente indistinguible y está compuesto por tres linajes evolutivos, considerándose $B$. pinnata como sinonimia de $B$. calliptera. Dada la gran diversidad morfológica de las especies de Bostrychia en la región del Pacífico colombiano, se ha considerado necesario considerar como especies crípticas a $B$. calliptera, B. pinnata, $B$. radicans y $B$. moritziana con base en los criterios taxonómicos clásicos y en las filogenias halladas en estudios recientes.

Las respuestas fisiológicas, como la actividad fotosintética frente a la desecación de las algas debida al ciclo mareal, son claves para explicar la distribución y la zonación de las especies en la zona intermareal. En la costa pacífica colombiana estas especies son claves por su contribución a la productividad primaria de estos ecosistemas costeros.

A partir del análisis realizado se plantean nuevos desafíos de investigación orientados, por un lado, a aspectos de la sistemática molecular, como la complementación de la filogenia del género Bostrychia y la filogeografía de las poblaciones del ensamblaje algal presentes en otros ambientes intermareales costeros del Pacífico, y por otro lado, a esclarecer los mecanismos de la tolerancia de las especies al estrés ambiental y el uso potencial de sus compuestos con actividad biológica para la descontaminación y biotratamiento de cuerpos de agua marinos costeros.

\section{Agradecimientos}

A la Universidad del Valle por al apoyo en el desarrollo académico y científico de la línea de ficología y al grupo de investigación en biología de plantas y microorganismos adscritos al programa de Biología. A los muchos estudiantes de pregrado y postgrado por el trabajo de campo y de laboratorio realizado en el marco de la construcción del semillero de ficología y de la colección de algas del Herbario de la Universidad (CUV). A Diego Hernández por su apoyo decisivo en los experimentos de manipulación de ADN y en el tratamiento de los datos de biología molecular. A Elizabeth Patiño por su apoyo permanente y en la edición del documento final.

\section{Conflicto de intereses}

El autor declara no tener conflicto de intereses.

\section{Referencias}

Abe, S., Kurashima, A., Yokohama, Y., Tanaka, J. 2001. The cellular ability of desiccation tolerance in Japanese intertidal seaweeds. Bot. Mar. 44: 125-131.

Ansell, R. 2013. The coexistence of cryptic algal species. Thesis for Master of Science Part II. p. 3-101.

Baos, R. Morales, S. 2007. Algas asociadas a un manglar en el Pacifico colombiano. Municipio de Buenaventura-Valle del Cauca. En: Facultad de Ciencias Agropecuarias. 5 (2): 84-89.

Bula-Meyer, G. 1989. Altas temperaturas estacionales del agua como condición disturbadora de las macroalgas del Parque Nacional Tayrona, Caribe colombiano: una hipótesis. An. Inst. Inv. Mar. Punta Benín. 19 (20): 9-21.

Burkholder, P.R. L.R. Almodóvar. 1973. Studies on mangrove algal communities in Puerto Rico. Florida Sci. 36: 50-66.

Dawes, C. 1996. Macroalga diversity, standing stock and productivity in northern mangal on the west coast of Florida. Nova Hedwigia, Beiheft. 112: 525-535.

Dreckmann, K. Sentíes, A. Pedroche, F. Callejas, M. 2006. Diagnóstico florístico de la ficología marina bentónica en Chiapas. Hidrobiol. 16 (2): 147-158.

Dinakar, C., Djilianov, D., Bartels, D. 2012. Photosynthesis in desiccation tolerant plants: Energy metabolism and antioxidative stress defense. Plant Sci. 182: 29-41.

Futuyma, D. 2009. Evolution. 2nd ed. Sinauer Associates, Sunderland, Massachusetts. USA. p. 632. 
Gavio, B., Fredericq, S. 2002. Grateloupia turuturu(Halymeniaceae, Rhodophyta) is the correct name of the nonnative species in the Atlantic known as Grateloupia doryphora. Eur. J. Phycol. 37: 349-359.

Gylle, A., C., Nygard, N.G., Ekelund. 2009. Desiccation and salinity effects on marine and brackish Fucus vesiculosus L. (Phaeophyceae). Phycologia. 48: 156-164.

Gianoli, E. 2004. Plasticidad fenotípica adaptativa en plantas. En: Cabrera, H. 2004. Fisiología ecológica en plantas. Mecanismos y respuestas en estrés en los ecosistemas. Ed. Universitarias de Valparaíso (Chile). p. 13-26.

Kamiya, M. West, J. A. 2014. Cryptic diversity in the euryhaline red alga Caloglossa ogasawaraensis (Delesseriaceae, Ceramiales). Phycologia. 53 (4): 374-382.

Kim, K.Y., D.J., Garbary. 2007. Photosynthesis in Codium fragile (Chlorophyta) from a Nova Scotia estuary: Responses to desiccation and hyposalinity. Mar. Biol. 151: 99-107.

Karsten, U., T. Sawall, Wiencke, C. 1998. A survey of the distribution of UV-absorbing substances in tropical macroalgae. Phycol. Res. 46: 271-279.

King, R. Puttock, C. 1994. Macroalgae associated with mangroves in Australia: Rhodophyta. Bot. Mar. 37: 181-191.

Lee, R. E. 2008. Phycology, 4th edition. University Press, Cambridge.

Leprince, O., Buitink, J. 2010. Desiccation tolerance: From genomics to the field. Plant Sci. 179: 554-564.

Lobban, Ch.S., Harrison, P.J. 1994. Seaweed Ecology and Physiology. Cambridge University Press. p. 366.

Markmann, M. Tautz, D. 2005. Reverse taxonomy: An approach towards determining the diversity of meiobenthic organisms based on ribosomal RNA signature sequences. Philos. Trans. R. Soc. Lond. B Biol. Sci. 360: 1917-1924.

Moore, J.P., Le, N.T., Brandt, W.F., Driouich, A., Farrant. 2009. Towards a systems-based understanding of plant desiccation tolerance. Trends Plant Sci. 14: 110-117.

Muangmai, N. West, J.A. Zuccarello, G.C. 2014. Evolution of four Southern Hemisphere Bostrychia (Rhodomelaceae, Rhodophyta) species: Phylogeny, species delimitation and divergence times. Phycologia. 53 (6): 593-601.

Murillo, M. Peña-Salamanca, E.J. 2013. Algas marinas bentónicas de la Isla Gorgona, costa pacífica colombiana. Rev. Biol. Trop. 62 (1): 27-41.

Peña-Salamanca, E.J., Zingmark R., Nietch C. 1999. Comparative photosynthesis of two species of intertidal epiphytic macroalgae on mangrove roots during submersion and emersion. J. Phycol. 35: 1206-1214.

Peña-Salamanca, EJ. 2008. Dinámica espacial y temporal de la biomasa algal asociada a las raíces de mangle en la bahía de Buenaventura, costa pacífica de Colombia. Bol. Invest. Mar. Cost. 37 (2): 55-70.

Peña-Salamanca, E.J., Cantera-Kintz, J., Silverstone-Sopkin, P.A. 2014. Mangrove forests of the Pacific coast of Colombia. En: Plants of the South American Pacific mangrove swamps: Colombia, Ecuador, Perú. Cornejo, X. (editor). Publicaciones del Herbario GUAY, Facultad de Ciencias Naturales de la Universidad de Guayaquil. Guayaquil, Ecuador. p. 15-20.

Peña-Salamanca, EJ. Marín-Salgado, H. 2016. Ficología aplicada: características, usos y cultivo de algas marinas. Colección: Ciencias Naturales y Exactas. Cali, Colombia. p. 13-153.
Post, E. 1936. Zur verbreitung y okologie der Bostrychia-Caloglossaassoziation. Int. Rev. Gesamten. Hydrobiol. 48: 47-152.

Rambaut A. 2009. FigTree v1.3.1: Tree figure drawing tool. Fecha de consulta: 4 de febrero, 2017. Disponible en: http://tree. bio.ed.ac.uk/software/figtree/

Rodríguez, C., Stoner, A.W. 1990. The epiphyte community of mangrove roots in a tropical estuary: Distribution and biomass. Aquat. Bot. 36: 117-126.

Saengkaew, J., Muangmai, N., Zuccarello, G.C. 2016b. Cryptic diversity of the mangrove-associated alga Bostrychia (Rhodomelaceae, Rhodophyta) from Thailand. Botanica Marina. 59: 363-372.

Saez, A.G., Lozano, E. 2005. Body doubles. Cryptic species: As we discover more examples of species that are morphologically indistinguishable, we need to ask why and how they exist. Nature. 433: 111.

Skene, K.R. 2004. Key differences in photosynthetic characteristics of nine species of intertidal macroalgae are related to their position on the shore. Can. J. Bot. 82: 177-184.

Schnetter, R., Bula-Meyer, G. 1982. Marine algen der Pazifkuste von Kolombien. Chlorophyceae, Phaeophyceae, Rhodophyceae. Bibliotheca. Phycol. 60: 1-287.

Sherwood, A.R. Zuccarello, G.C. 2016. Chapter 8. Phylogeography of Tropical Pacific marine algae. In: Seaweed Phylogeography: Adaptation and evolution of seaweeds under environmental change. Zi-Min $\mathrm{Hu} \&$ Ceridwen Fraser (Ed). Springer Netherlands. p. 211-226.

Tanaka, J. Chihara, M. 1987. Species composition and vertical distribution of macroalgae in brackish waters of Japanese mangrove forest. Bull. Natn. Sci. Mus. Tokyo. Ser. B. 13: 141-150.

Yarish, C., Kraemer, G., Kim, J., Carmona, R., Neefus, C., Nardi, G., Curtis, J., Pereira, R., Rawson, M. 2004. The bioremediation potential of economically important seaweed in integrated aquaculture systems with finfish. The 33rd UJNR aquaculture Panel Symposium: "Ecosystem and carrying capacity of aquaculture ground". Japan. NRIA, Hiroshima University, Kagishima University and SNFRI. p. 32-33.

Yang, E., Boo, S., Bhattacharya, D., Saunders, G., Knoll, A., Frederiq, S., Yoon H. 2016. Divergence time estimates and the evolution of major lineages in the florideophyte red algae. Scientific reports. 6: 1-11.

Zuccarello, G.C., J.A West, R., King. 1999b. Evolutionary divergence in the Bostrychia moritziana / B. radicans complex (Rhodomelaceae, Rhodophyta): Molecular and hybridization data. Phycologia. 38 (3): 234-244.

Zuccarello, G.C., J.A West. 2002. Phylogeography of Bostrychia calliptera - B. pinnata complex (Rhodomelaceae, Rhodophyta) and divergence rates based on nuclear, mitochondrial and plastid DNA markers. Phycologia. 41: 49-60.

Zuccarello, G.C., J.AWest. 2003. Multiple cryptic species: Molecular diversity and reproductive isolation in the Bostrychia radicans / B. moritziana complex (Rhodomelaceae, Rhodophyta) with focus on North American isolates. J. Phycol. 39: 948-59.

Zuccarello, G.C. J.A. West. 2011. Insights into evolution and speciation in the red alga Bostrychia: 15 years of research. Algae. 26 (1): 3-14. 